\title{
Analysis of Multiphase Fluid Flow, Heat, Mass and Charge Transfer inside Electrochemical Systems
}

\author{
Martin Desilets \\ Sherbrooke University, Canada
}

The development of new modeling tools and the increase of CPU power now permit the assessment and optimization of complex industrial applications. In this presentation, it is shown how advanced multi-physics modeling is used to study new designs and operating conditions to reduce energy consumption, increase productivity and lifetime of electrochemical systems. Three examples are given to illustrate the application of these models to systems that cover the full range of electrochemical applications from energy storage devices (batteries) to molten salt electrolysis cells.

The first example is focussed on the dynamic modeling of lithium-ion batteries (LIB) to assess the impact of electrode aging on its charge-discharge behavior. LIB physics-based models that rest on engineering governing equations involve the identification of many dimensional and physico-chemical parameters. A performant parameter identification methodology is used to identify the impact of operating conditions and aging on most sensitive parameters. In the second example, finite element models that are typically used to improve the thermoelectric design of Hall-Héroult cells for aluminium production are presented. State-of-the-art models are now able to represent coupled phenomena including transient heat transfer with radiation and phase change, moving boundaries, degrading materials and anode consumption. These advanced tools are exploited to study the impact of industrial operations on the energy consumption of such reactors. The last example will show the challenges posed by the development of a recent two-phase flow model representing the behavior of a lithium production cell considering convection, diffusion and migration mass transport phenomena. This model has been used to find design solutions in order to reduce the specific energy of this molten salt electrolysis cell.

Future perspectives about the implementation of advanced engineering models into new industrial practices, for diagnostic and control purposes, will conclude the presentation. 